\title{
Dispersal Kernel Determines Symmetry of Spread and Geographical Range for an Insect
}

\author{
Jeffrey D. Holland ${ }^{1,2}$ \\ ${ }^{1}$ Department of Entomology, Purdue University, 901 W. State St., West Lafayette, IN 47907, USA \\ ${ }^{2}$ The Purdue Interdisciplinary Center for Ecological Sustainability, Purdue University, 901 W. State St., \\ West Lafayette, IN 47907, USA
}

Correspondence should be addressed to Jeffrey D. Holland, jdhollan@purdue.edu

Received 8 April 2009; Accepted 10 November 2009

Recommended by Madhur Anand

The distance from a source patch that dispersing insects reach depends on the number of dispersers, or random draws from a probability density function called a dispersal kernel, and the shape of that kernel. This can cause asymmetrical dispersal between habitat patches that produce different numbers of dispersers. Spatial distributions based on these dynamics can explain several ecological patterns including megapopulations and geographic range boundaries. I hypothesized that a locally extirpated longhorned beetle, the sugar maple borer, has a new geographical range shaped primarily by probabilistic dispersal distances. I used data on occurrence from Ontario, Canada to construct a model of geographical range in Indiana, USA based on maximum dispersal distance scaled by habitat area. This model predicted the new range boundary within $500 \mathrm{~m}$ very accurately. This beetle may be an ideal organism for exploring spatial dynamics driven by dispersal.

Copyright (C) 2009 Jeffrey D. Holland. This is an open access article distributed under the Creative Commons Attribution License, which permits unrestricted use, distribution, and reproduction in any medium, provided the original work is properly cited.

\section{Introduction}

Displacement of many animals, including insects, is predicted by random walks even if the actual path deviates from Brownian-type motion [1]. The distance that individuals travel can be described as random draws from a probability density function termed a dispersal kernel. It follows that the maximum distance at which potential colonists reach habitat patches will scale with the number of dispersers leaving a given patch. This number is often assumed to scale with size of the source habitat patch [2,3]. The left-heavy shape of many dispersal kernels leads to a more or less welldefined ring that defines the area of likely colonization, as in Figure 4 of Ovaskainen [4]. While the sharpness of the outer ring edge will be determined by the shape of the dispersal kernel; diffusion at larger distances from the source patch will further sharpen this boundary.

Larger habitat patches will not only be more likely to send colonists farther than smaller patches, they are also less likely to experience local extinction if they support larger populations [5]. Larger patches will act as more persistent sources of potential colonists than smaller patches (e.g., [6]) further reinforcing the probabilistic scaling of maximum successful dispersal distance around different sized patches. These hypothesized spatial dynamics lead to a view of occupancy dynamics that is neither classic metapopulation nor sourcesink in nature, but can be visualized as a cascade of dispersers with the distances reached scaled by source size. This has the potential to explain asymmetrical dispersal between habitat patches as well as geographical range boundaries for some species. It may also explain megapopulation, or interacting collections of metapopulations, structures observed in some animals $[7,8]$. I term this heuristic view of spatial dynamics a cascading megapopulation model.

Glycobius speciosus (Say), the sugar maple borer (Coleoptera: Cerambycidae) has been extirpated from a large portion of the American Midwest within its former range [9]. The larvae develop over two years in the cambial layer of sugar maple trees. This leads to partial or complete girdling of the conductive tissue of the tree. While very little is known about the dispersal of this beetle, the resultant scars indicate where the species has occurred in the recent past. This beetle is an ideal species to test the cascading megapopulation model because it leaves long-lasting scars that are very easily 
found and recognized on attacked trees [10]; it usually exists at densities below that of many insects [9] potentially making it susceptible to local extinctions, and a current area of extirpation is near the center of its former range and so is likely not caused by environmental limits common at range boundaries [11]. I hypothesized that G. speciosus has been extirpated from part of its range by forest fragmentation and that it exists as a cascading megapopulation as described above. I therefore predicted that this heuristic model would accurately predict the new range boundary.

\section{Methods}

I used survey data on forest trees and scars from $G$. speciosus from the Ottawa region in Ontario Canada to parameterize a cascading megapopulation model. This was used in ArcView GIS (ESRI, Redlands, USA) to predict the new range boundary across Indiana, USA. Forests were then surveyed both inside and outside the predicted boundary to validate the model.

2.1. Generating the Model. Plots measuring $25 \mathrm{~m}$ by $12.5 \mathrm{~m}$ within forests in the Ottawa region had previously had data on tree species and size recorded [12]. The 107 plots containing mature sugar maple trees $(\mathrm{dbh}>10 \mathrm{~cm})$ were surveyed for the larval scars from G. speciosus. Area of surveyed forest patches, and edge-to-edge distance to the nearest neighboring forest patch of at least $8,16,32, \ldots, 32,768$ ha (doubling series), was previously measured as part of a separate study of extinction thresholds [13]. For each size class, a logistic regression of scar presence/absence and distance from the nearest forest patch in the size class were conducted. Forest patches were weighted by the square root of the number of plots they contained. Lowest-log (likelihood) was used to select the regressions indicating forests that were operating as sources of colonists that were indicated by patches around them being occupied out to some threshold distance beyond which patches tend to be unoccupied. Overlapping size classes may seem artificial, but it avoids a potential problem with multiple potential source patches. If patch 1 in Figure 1 was occupied, using nonoverlapping size classes could lead to the conclusion that colonists had originated from patch 2. This could inflate the likely dispersal distance. Using overlapping size classes does lead to correlations between the different size-distance regressions. I therefore only included in the final model those source forest sizes that were both significant and not significantly correlated to other included sizes. Tables of area under the receiver-operator characteristic curve (AUC) were used to identify the threshold distances separating colonized and unreached forest patches around these source forests. The identified forest size classes and dispersal distances around them became the basis of the cascading megapopulation model in GIS used to predict the new range boundary in Indiana.

To compare the cascading megapopulation model prediction to simpler alternate models, I also used similar methods to identify forest sizes and dispersal distances based on the Ottawa data to create geographical range predictions

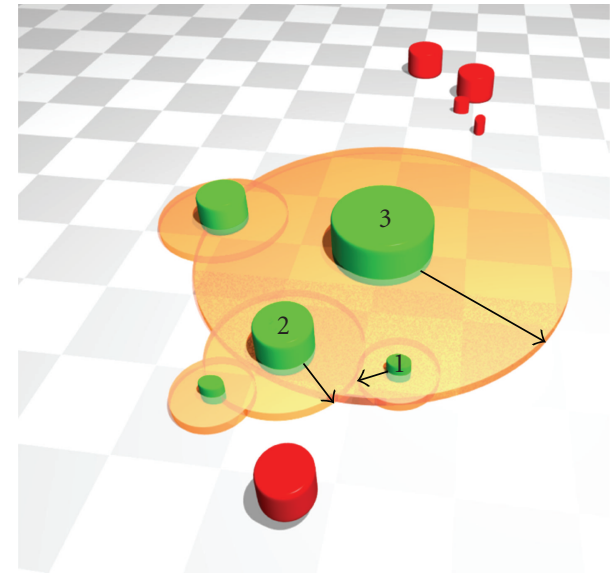

FIgure 1: Hypothetical landscape with occupied (green) and unoccupied (red) forest patches in an agricultural matrix. Orange rings around occupied patches cover area likely to receive colonists. Arrows indicate threshold distance between surrounding occupied and unoccupied habitat patches. Geographical range is the union of these rings and all forest patches they intersect.

based upon: (1) a minimum forest size requirement, (2) a fixed maximum dispersal distance, that is, this distance did not increase with forest size and more draws from the dispersal kernel, and (3) the same model as the cascading megapopulation but with non-overlapping forest size classes.

2.2. Predicting and Validating the New Geographical Range Boundary of G. speciosus. I first tested whether the identified threshold distance between occupied and unoccupied forest patches around potential sources scaled with source size using a linear regression. To predict the new geographical range of G. speciosus in Indiana, I extracted all forested cells from the 1992 Indiana National Land Cover Data [14] and calculated the area of contiguous forest patches. For the cascading megapopulation model, I predicted the occupied range sequentially. All forests in the largest size class that were previously found to act as likely sources were identified and buffered by the threshold dispersal distance for that size class. All forest patches in the next smaller size class that intersected this buffer were then included and in turn buffered by their identified threshold dispersal distance. This was repeated until all size classes were included and no further potential source forests were included. The new geographical range was then predicted to be the union of all included forests, their buffers, and any forests intersecting those buffers.

I validated the new range models by surveying forests for G. speciosus scars on mature maple trees on both sides of the range boundary in Indiana during 2006 and 2007, after the prediction had been made. I surveyed for scars in 23 forests. If I did not at first find scars, I continued to survey until I had exhausted the likelihood of a false negative, usually examining well over 100 mature sugar maple trees during a period of more than an hour. Given that the beetle attacks approximately a third of the maples present in forests where the beetle occurs $[10,15]$, finding this many unaffected trees 
TABLE 1: Confusion matrix for cascading megapopulation with overlapping size ranges and alternative models for geographical range of $G$. speciosus. Overlap refers to overlap in forest size classes. CM: cascading megapopulation; MA: minimum area; pres.: presence; abs: absence.

\begin{tabular}{lcccc}
\hline Model & Correctly Predicted pres & Correctly Predicted abs & Unpred pres & Unpred abs \\
\hline CM with overlap & 15 & 8 & 0 & 0 \\
CM no overlap & 15 & 3 & 0 & 5 \\
MA with overlap & 14 & 3 & 1 & 5 \\
MA no overlap & 14 & 3 & 1 & 5 \\
\hline
\end{tabular}

sequentially when the beetle has been present is extremely unlikely [9]. Surveying both sides of the predicted boundary in new locations, even with a small number of sites, is a strong test of the model [16]. To compare the models I constructed a confusion matrix with results of all model predictions.

\section{Results}

The distance separating occupied and unoccupied forests around potential source forests did scale with source forest size. The initial logistic regressions of occupancy against distance from source were significant at the 0.05 level for 10 of the 13 source sizes using overlapping size classes. A linear regression of threshold distance versus log (source area) using these 10 source sizes was significant $(d f=9$, $F=11.27, P=.01, R^{2}=0.77$ ) with a positive slope. The models based on a maximum isolation distance were therefore not considered further. Logistic regression analyses of the Ottawa data on occurrence showed that G. speciosus scars were almost never found in forest patches containing sugar maple trees if the patch was farther than $2489 \mathrm{~m}$ from a forest patch $\geq 1024$ ha $\left(d f=1, \chi^{2}=10.15, P=.0014\right.$, AUC $=$ 0.912 ), or farther than $4753 \mathrm{~m}$ from a forest patch $\geq 16384$ ha $\left(d f=1, \chi^{2}=4.92, P=.0266, \mathrm{AUC}=0.763\right)$. Distances to all other forest size classes were correlated to the distances to one of these two forest sizes and therefore not used in the cascading megapopulation model. The geographical range prediction was therefore created by buffering $4800 \mathrm{~m}$ around forests $\geq 16384 \mathrm{ha}$, and adding all forests $\geq 1024$ ha that intersected this buffer, and then buffering the $\geq 1024$ ha forests by $2500 \mathrm{~m}$. The predicted range then included all forests intersecting the union of these buffers and any additional intersected forest patches.

I surveyed 23 forests for G. speciosus scars, eight outside the predicted boundary and 15 within the boundary. If scars were not initially found, I surveyed until at least 100 large maple trees had been checked for scars. All surveyed forests contained abundant mature sugar maple trees. The surveys were $100 \%$ in agreement with the range prediction (Table 1) even with some of the sites quite close to the predicted range boundary, for example, within $500 \mathrm{~m}$ (Figure 2). Several additional sites were also surveyed but were close to forests already surveyed (with a few $\mathrm{km}$ ) so they are not included as separate replicates. These omitted sites were also always in agreement with the prediction. Several forests without $G$. speciosus were larger than the 1024 ha size that are apparently consistently occupied and a source of colonists within the range boundary. The other alternative models did not fit

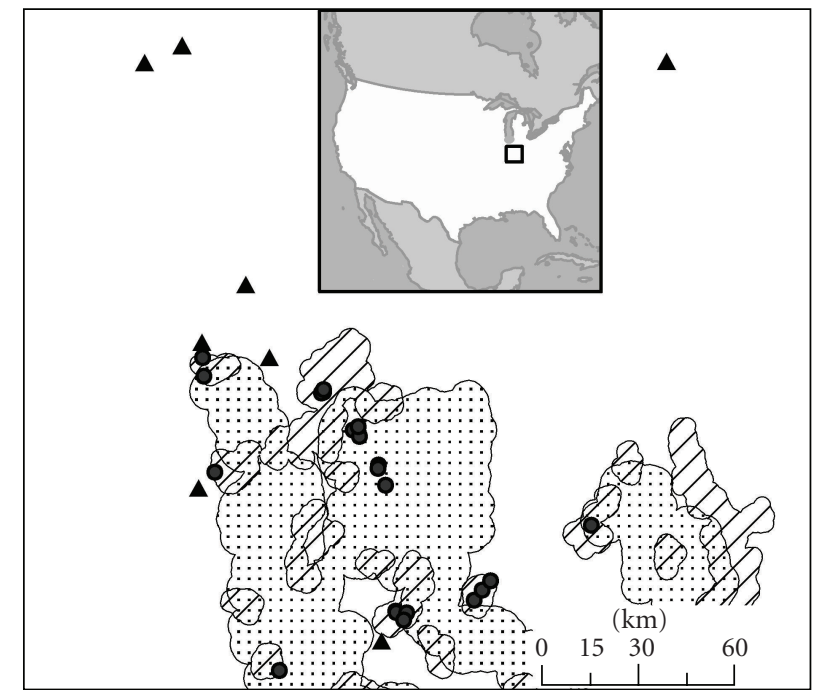

Figure 2: Map of southern Indiana, U.S.A. showing results of surveys in area of predicted new range boundary. Stippled and diagonal-hatched areas are predicted range; stippled, $4,800 \mathrm{~m}$ from 16,384 ha forest; diagonal-hatch, 2,500 $\mathrm{m}$ from 1,024 ha forest that intersect cross-hatched area; circles, larval scars detected; triangles, larval scars not detected. Inset: study area within Indiana, USA.

the validation survey results nearly as well as the cascading megapopulation model (Table 1).

\section{Discussion}

The threshold distance between occupied and unoccupied patches did scale with source patch size, indicating that using a probabilistic model of colonization and occurrence is more appropriate than assuming a specific dispersal distance. The fit of the cascading megapopulation model was much better than the models based on either a minimum area or cascading model with non-overlapping size ranges. To ensure that the discarded maximum dispersal distance model (created with both overlapping and non-overlapping forest size ranges) would not have also provided a good prediction; this was tested $a$ posteriori as well. These models fared worse than the others.

The heuristic cascading megapopulation model allows researchers to use knowledge of the dispersal kernel or empirical threshold distances plus either population sizes or patch sizes to scale up from metapopulation dynamics to the geographical range. This will work better for some species than others. Additional parameters such as area or radius of 
target patches may be necessary for predicting occupancy of other taxa. How immigration is dependent on target patch size is likely partially determined by whether dispersal occurs near the ground or at higher altitude, whether dispersers engage in searching during dispersal, and whether dispersers are able to detect habitat and orient towards it at a distance [17]. It is also an unusual species that responds to the landscape in a binary habitat-matrix way [18].

This model of occupancy implies that asymmetrical dispersal between patches can occur in the absence of differences in habitat quality. If patches are located farther apart than the likely probabilistic maximum dispersal around the smaller of a pair of patches, such asymmetry can result [19].

The sugar maple borer has the potential to be a model species for studying large scale spatial population dynamics. The long lasting scars prevent year-to-year changes in occupancy from causing the apparent range boundary from shifting at these trivial temporal scales. While I cannot be certain that forests with recent scars are occupied the year they are surveyed, the longer temporal scale indicated by these scars is more amenable to large scale occupancy studies. Large networks of forests with suitable host trees and some large forest patches present within the area of extirpation suggest that this species exists as a megapopulation, or a collection of metapopulations, any of which may become completely unoccupied [7].

\section{Acknowledgments}

The author thanks C. J. Foley, D. Ladd, G. Murray, J. C. Rowland, and M. J. Skvarla for fieldwork help. D. C. Allen and K. B. Adams helped him understand G. speciosus biology and offered encouragement throughout this project. J. Shukle helped with the figures. This paper was improved by comments from C. J. Foley, K. Raje, H. I. Rowe, C. S. Sadof, R. K. Swihart, and two anonymous reviewers.

\section{References}

[1] A. Okubo and P. Kareiva, "Some examples of animal diffusion," in Diffusion and Ecological Problems: Modern Perspectives, A. Okubo and S. A. Levin, Eds., pp. 170-196, Springer, New York, NY, USA, 2001.

[2] R. H. MacArthur and E. O. Wilson, The Theory of Island Biogeography, Princeton University Press, Princeton, NJ, USA, 1967.

[3] I. Hanski, "A practical model of metapopulation dynamics," Journal of Animal Ecology, vol. 63, no. 1, pp. 151-162, 1994.

[4] O. Ovaskainen, "Habitat-specific movement parameters estimated using mark-recapture data and a diffusion model," Ecology, vol. 85, no. 1, pp. 242-257, 2004.

[5] S. L. Pimm, H. L. Jones, and J. Diamond, "On the risks of extinction," American Naturalist, vol. 132, no. 6, pp. 757-785, 1988.

[6] V. M. Stevens and M. Baguette, "Importance of habitat quality and landscape connectivity for the persistence of endangered natterjack toads," Conservation Biology, vol. 22, no. 5, pp. 1194-1204, 2008.
[7] R. E. Ricklefs, "The megapopulation: a model of demographic coupling between migrant and resident land bird populations," in Ecology and Conservation of Neotropical Migrant Land-Birds, J. M. Hagan III and D. W. Johnston, Eds., pp. 537548, Smithsonian Institution Press, Washington, DC, USA, 1992.

[8] R. J. Wilson, S. Ellis, J. S. Baker, M. E. Lineham, R. W. Whitehead, and C. D. Thomas, "Large-scale patterns of distribution and persistence at the range margins of a butterfly," Ecology, vol. 83, no. 12, pp. 3357-3368, 2002.

[9] J. D. Holland, "Glycobius speciosus (Say) (Coleoptera: Cerambycidae) has been extirpated from much of Midwestern USA," The Coleopterists Bulletin, vol. 63, no. 1, pp. 54-61, 2009.

[10] W. G. Newton and D. C. Allen, "Characteristics of trees damaged by sugar maple borer, Glycobius speciosus (Say)," Canadian Journal of Forest Research, vol. 12, no. 4, pp. 738744, 1982.

[11] J. H. Lawton, S. Nee, A. J. Letcher, and P. H. Harvey, "Animal distributions: patterns and processes," in Large-Scale Ecology and Conservation Biology, P. J. Edwards, R. M. May, and N. R. Webb, Eds., pp. 41-58, Blackwell, Oxford, UK, 1994.

[12] A. T. Darwin, D. Ladd, R. Galdins, T. A. Contreras, and L. Fahrig, "Response of forest understory vegetation to a major ice storm," Journal of the Torrey Botanical Society, vol. 131, no. 1, pp. 45-52, 2004.

[13] J. D. Holland, L. Fahrig, and N. Cappuccino, "Fecundity determines the extinction threshold in a Canadian assemblage of longhorned beetles (Coleoptera: Cerambycidae)," Journal of Insect Conservation, vol. 9, no. 2, pp. 109-119, 2005.

[14] J. E. Vogelmann, S. M. Howard, L. Yang, C. R. Larson, B. K. Wylie, and N. Van Driel, "Completion of the 1990s national land cover data set for the conterminous United States from landsat thematic mapper data and ancillary data sources," Photogrammetric Engineering and Remote Sensing, vol. 67, no. 6, pp. 650-662, 2001.

[15] A. L. Shigo, W. B. Leak, and S. M. Filip, "Sugar-maple borer injury in four hardwood stands in New Hampshire," Canadian Journal of Forest Research, vol. 3, no. 4, pp. 512-515, 1973.

[16] R. G. Pearson, C. J. Raxworthy, M. Nakamura, and A. T. Peterson, "Predicting species distributions from small numbers of occurrence records: a test case using cryptic geckos in Madagascar," Journal of Biogeography, vol. 34, no. 1, pp. 102-117, 2007.

[17] J. Bowman, N. Cappuccino, and L. Fahrig, "Patch size and population density: the effect of immigration behavior," Ecology and Society, vol. 6, no. 1, 2002.

[18] H. T. Murphy and J. Lovett-Doust, "Context and connectivity in plant metapopulations and landscape mosaics: does the matrix matter?" Oikos, vol. 105, no. 1, pp. 3-14, 2004.

[19] T. J. Kawecki, "Ecological and evolutionary consequences of source-sink population dynamics," in Ecology, Genetics, and Evolution of Metapopulations, I. Hanski and O. E. Gaggiotti, Eds., pp. 387-414, Elsevier Academic Press, Burlington, Mass, USA, 2004. 

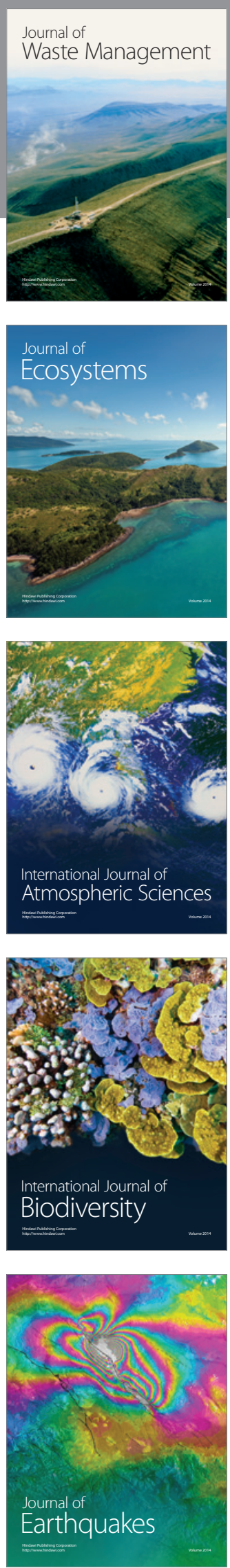
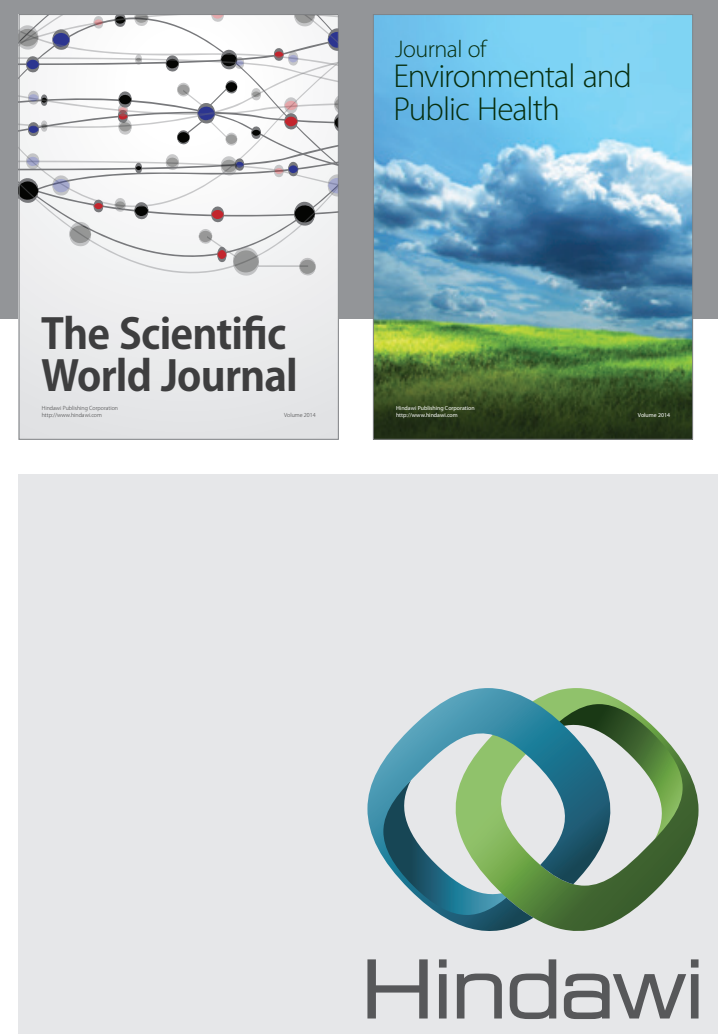

Submit your manuscripts at

http://www.hindawi.com
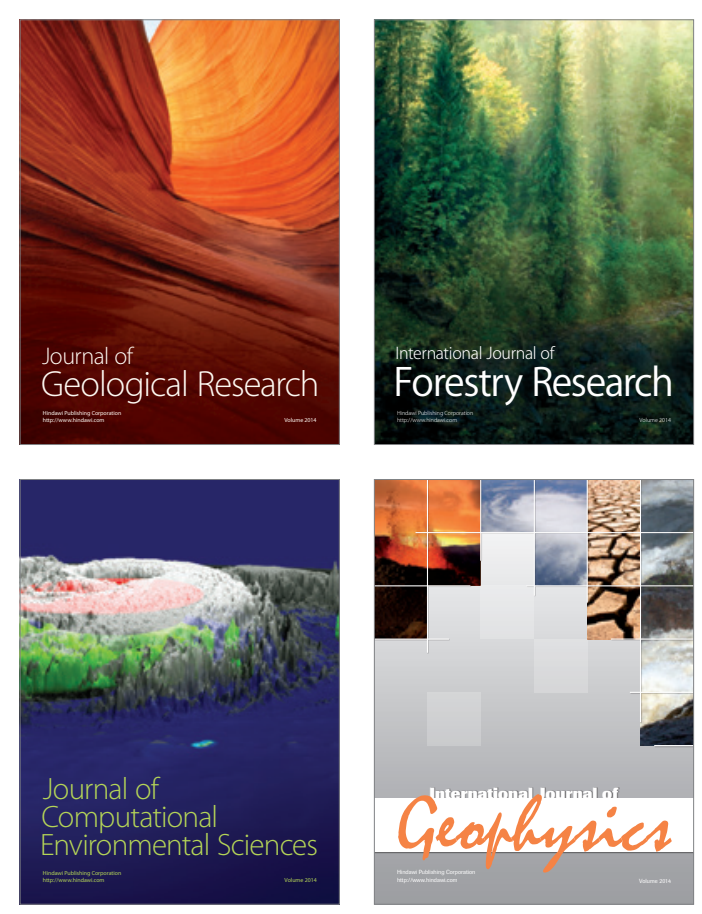
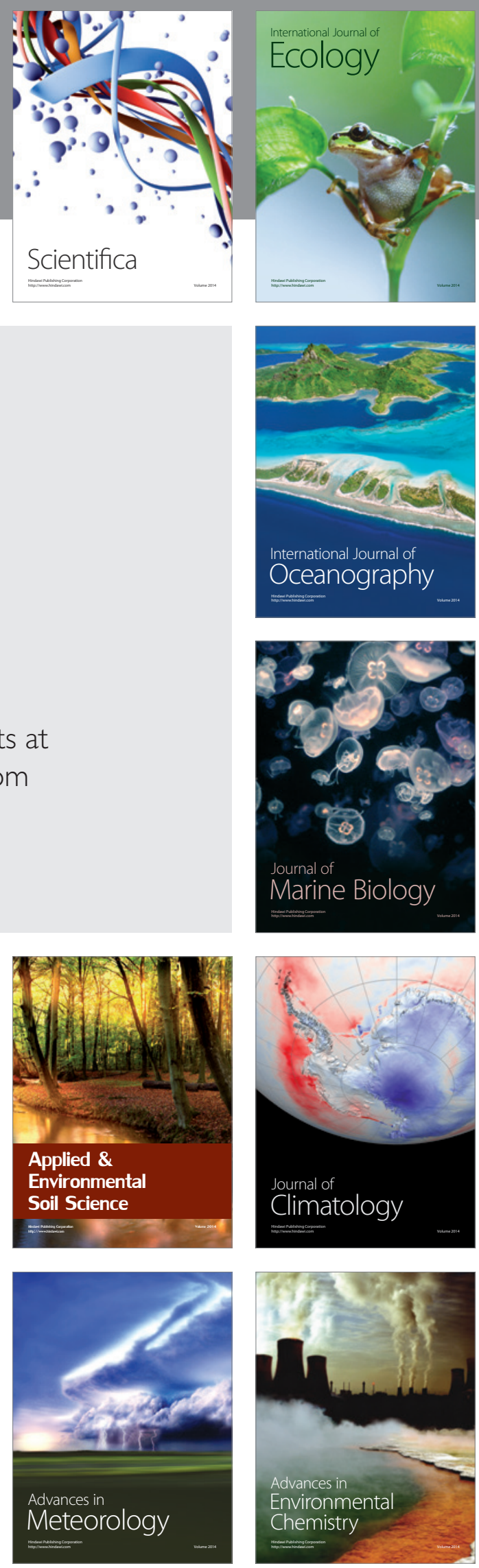